\title{
Die deutsche EU-Ratspräsidentschaft 2020: selektive Föderalisierung des Integrationsprozesses
}

\author{
Katrin Böttger und Mathias Jopp*
}

\begin{abstract}
This article analyses the most important issues and decisions of the German EU Council Presidency, but also its "leftovers". Almost beyond expectations, the German Government proved to be a successful mediator and honest broker for viable compromises in a number of conflicting areas. It was possible, in the second half of 2020, to resolve basic EU financing issues for the next seven years period, prevent a no-deal Brexit through a trade agreement with the United Kingdom, conclude an investment agreement with China and achieve the adoption of a rule of law mechanism against corruption in the use of EU funds by national actors. During the German Presidency, once again in European integration, a centralization of important tasks at the Brussels level can be identified, combined with a partial strengthening of supranational institutions.
\end{abstract}

Nach 2007 hatte Deutschland vom 1. Juli bis 31. Dezember 2020 erstmals wieder den Vorsitz im Rat der Europäischen Union (EU) inne. Aufgrund der Erfolge $2007^{1}$ und der herausgehobenen Rolle Deutschlands als größter EU-Mitgliedstaat werden deutsche EU-Ratspräsidentschaften immer mit besonderer Aufmerksamkeit verfolgt. Auch die Erwartungen an die Ratspräsidentschaft 2020, in wichtigen Politikbereichen Kompromisse zu finden, Pakete $\mathrm{zu}$ schnüren und die europäische Integration voranzutreiben, waren besonders groß. ${ }^{2}$ In diesem Beitrag möchten wir nachzeichnen, wie Deutschland die Ratspräsidentschaft vorbereitet hat, wie die Entwicklungen in der ersten Jahreshälfte 2020 insbesondere mit Blick auf die Ausbreitung des Coronavirus und die Auswirkungen auf die Ratspräsidentschaft verlaufen sind und was die wichtigsten Themen sowie Beschlüsse der deutschen Ratspräsidentschaft, aber auch ihre „Leftovers“ waren, um dann eine erste Bewertung der Ergebnisse vorzunehmen. Hierbei setzte sich unseres Erachtens der seit 2016 wieder stärker gewordene Trend zur Vergemeinschaftung und Supranationalisierung in der deutschen Europapolitik, einschließlich des übergeordneten Interesses an der Erhaltung des europäischen Einigungswerkes sowie des Zusammenhalts der EU-27, fort. ${ }^{3}$ Auch wenn Deutschland mit den nicht immer einfachen Gruppenbildungen unter den EU-Mitgliedstaaten umgehen und zwischen ihnen vermitteln musste, ${ }^{4}$ hat die deutsche Ratspräsidentschaft

* Dr. Katrin Böttger, Direktorin am Institut für Europäische Politik, Berlin.

Prof. Dr. Mathias Jopp, ehemaliger Direktor des Instituts für Europäische Politik, Berlin; Honorarprofessor der Universität Passau und der Eberhard Karls Universität Tübingen; Moduldirektor für European Studies, Centre international de formation européenne (CIFE), Nizza/Berlin.

Stand des Beitrags ist der 11. Januar 2021. Die AutorInnen danken Lennart Belke für seine vielfältige Zuarbeit und Unterstützung.

1 Daniel Göler/Mathias Jopp: Kann Europa gelingen? Vorhaben und Chancen der deutschen Ratspräsidentschaft, in: integration 1/2007, S. 3-24; Daniel Göler/Mathias Jopp: Eine Präsidentschaft in einer anderen Zeit: die deutsche Ratspräsidentschaft 2020 im Vergleich zu 2007, in: integration 3/2020, S. 206-207.

2 Katrin Böttger/Funda Tekin: Germany's EU Council Presidency 2020 - Achieving more than managing expectations in challenging times, Institut für Europäische Politik: Berlin Perspectives 1/2020.

3 Katrin Böttger/Mathias Jopp: Grundlinien deutscher Europapolitik, in: Katrin Böttger/Mathias Jopp (Hrsg.): Handbuch zur deutschen Europapolitik, Baden-Baden 2021, S. 13-30.

4 Kai-Olaf Lang/Nicolai von Ondarza: Friends in Need. The Corona Pandemic Changes the Landscape of Groups and Coalitions in the EU, Stiftung Wissenschaft und Politik: SWP Comment 26/2020. 
trotz der Einschränkungen durch die COVID-19-Pandemie erneut auch in Deutschland zu einem intensiven, ressortübergreifenden Austausch und einer damit einhergehenden Sozialisierung und Europäisierung geführt. Dies unterstreicht die Relevanz, welche die nationalen Ratspräsidentschaften für das europäische Integrationsprojekt auch seit dem Vertrag von Lissabon weiterhin haben.

\section{Vom Erwartungs- zum Krisenmanagement}

Schon im Jahr 2019 begannen die Vorbereitungen für die deutsche EU-Ratspräsidentschaft im zweiten Halbjahr 2020. Diese standen zunächst unter dem Motto einer dezentralen Präsidentschaft, in der die einzelnen Ministerien Prioritäten, wichtige Treffen und Veranstaltungen sowie anstehende Entscheidungen identifizieren sollten. Während die Erwartungen an die deutsche Ratspräsidentschaft von außen, d.h. sowohl von der Zivilgesellschaft in Deutschland als auch von den anderen EU-Mitgliedstaaten, hoch waren, betrieb die Bundesregierung schon frühzeitig Erwartungsmanagement und unterstrich, dass es aller Erfahrung nach besser sei, die zu behandelnden Themen auf sich zukommen zu lassen und nicht zu viele Pläne vorab zu entwickeln. Diese Vorhersage hätte sich angesichts der COVID-19-Pandemie wohl nicht stärker bewahrheiten können. So betonte Angela Merkel bereits Ende April 2020 die Notwendigkeit, die Ziele der deutschen Ratspräsidentschaft anzupassen. ${ }^{5}$ Es zeichnete sich insbesondere ab, dass persönliche Treffen nicht oder nur in viel geringerem Umfang möglich sein würden. Tatsächlich mussten im Laufe der Ratspräsidentschaft zahlreiche geplante physische Treffen aufgrund von Corona-Fällen oder verhängten Quarantänemaßnahmen verschoben werden oder nicht alle VertreterInnen der Mitgliedstaaten konnten an anberaumten Sitzungen teilnehmen. Wiederholt mussten sich selbst Staats- und Regierungschefs untereinander vertreten. Angesichts dieser Lage war die Rolle des Ausschusses der Ständigen Vertreter (AStV) unter der Leitung der deutschen Ratspräsidentschaft und insbesondere des Botschafters Michael Clauß besonders bedeutend, da sich seine Mitglieder regelmäßig noch persönlich trafen. Weitere Rahmenbedingungen für die deutsche Ratspräsidentschaft waren eine noch nicht eingespielte Europäische Kommission, eine zuweilen konflikthafte Arbeitsteilung zwischen dem Präsidenten des Europäischen Rates Charles Michel und der Kommissionspräsidentin Ursula von der Leyen sowie ein heterogenes und unerfahrenes Europäisches Parlament. Nachdem in der Anfangsphase der Reaktion auf die COVID-19-Pandemie nationale Reflexe griffen, nahm die gegenseitige Unterstützung der Mitgliedstaaten schnell zu. Das Motto der deutschen Ratspräsidentschaft lautete dementsprechend „Gemeinsam. Europa wieder stark machen". ${ }^{\text {" }}$

5 Einleitende Aufzeichnung für einen Beschluss der EuropastaatssekretärInnen zu Auswirkungen der CoronaKrise auf die deutsche EU-Ratspräsidentschaft.

6 Auswärtiges Amt: Deutsche Präsidentschaft im Rat der Europäischen Union, abrufbar unter: https://www.eu20 20.de/ (letzter Zugriff: 28.1.2021). 


\section{Innere Reformpolitiken der Ratspräsidentschaft}

\section{Finanzrahmen und Aufbaufonds}

Der erste große Erfolg der deutschen Ratspräsidentschaft bestand in der Annahme des mehrjährigen Finanzrahmens (MFR) für die Jahre 2021 bis 2027 und des europäischen Aufbaufonds „Next Generation EU“ (NGEU) mit einem Gesamtvolumen von über 1,8 Billionen Euro auf dem Sondergipfel des Europäischen Rates vom 17. bis 21. Juli 2020. Dies war nicht nur einer der beiden längsten Europäischen Räte überhaupt, sondern es war auch die größte Summe, die haushaltspolitisch je im Rahmen der EU verhandelt wurde. ${ }^{7}$ Das Ergebnis war auch deshalb besonders bemerkenswert, weil die deutsche Bundesregierung erstmalig einer umfangreichen Geldaufnahme der EU am Kapitalmarkt zustimmte, nachdem während der Krise in der Eurozone durch die Finanz- und Staatsschuldenkrise genau dies von deutscher Seite immer abgelehnt worden war. ${ }^{8}$ Nun aber blieb angesichts des gewaltigen Einbruchs der europäischen Wirtschaft durch die Corona-Krise und angesichts der Belastungen durch die ergriffenen nationalen Konjunkturmaßnahmen kaum noch eine andere Möglichkeit, als einer Art Eurobonds für ein europäisches „Konjunkturpaket“ zuzustimmen, um Lasten und Risiken zu verteilen.

Die freilich schwierigen Verhandlungen über das EU-Finanzpaket wurden flexibel und pragmatisch von der deutschen Ratspräsidentschaft schon im Vorfeld der eigentlichen Gipfelentscheidungen wegweisend eingefädelt. Die südlichen EU-Mitgliedstaaten hatten unter Führung Italiens im März 2020 in einem Brief an den Präsidenten des Europäischen Rates, der von neun Mitgliedstaaten einschließlich Frankreich unterzeichnet worden war, eine gemeinschaftliche Schuldenaufnahme an den Kreditmärkten gefordert. ${ }^{9}$ Für die deutsche Bundesregierung, die zu diesem Zeitpunkt wie die Mehrheit im Europäischen Rat nur eine Aufbaufinanzierung aus dem Europäischen Stabilitätsmechanismus (ESM) wollte, war die Unterzeichnung des Briefs der Neun durch die französische Seite alarmierend, zumal das Vereinigte Königreich kein Mitspieler mehr im eigenen Lager war und die Gefahr bestand, dass die EU im Schatten der Eurokrise unter der Wucht der Pandemiefolgen auseinanderbrechen könnte.

Nachdem nicht nur östliche Mitgliedstaaten auf wesentlich mehr Ausgaben der EU drängten, sondern am 8. Mai 2020 auch das französische Finanzministerium hohe zusätzliche und auf drei Jahre befristete Mittel im EU-Haushalt vorgeschlagen hatte, ${ }^{10}$ vollzog Bundeskanzlerin Merkel eine entscheidende Wende und ging auf Frankreich und die südlichen Mitgliedstaaten zu. Am 18. Mai 2020 schlug sie gemeinsam mit Emmanuel Macron auf einer Pressekonferenz einen Aufbaufonds von 500 Milliarden Euro vor, der als Bestandteil des EU-Haushalts zusätzliche Mittel für die Überwindung der Pandemiefolgen,

7 Peter Becker: Nach dem EU-Gipfel: Historische Integrationsschritte unter Zeitdruck, Stiftung Wissenschaft und Politik: Kurz gesagt, 23. Juli 2020; und Peter Becker: Der Haushalt der EU als Chance in der Krise, Stiftung Wissenschaft und Politik: SWP-Aktuell 56/2020.

8 Katharina Gnath/Lucas Guttenberg/Nils Redeker: Deutschland und die Stabilität der Wirtschafts- und Währungsunion, in: Katrin Böttger/Mathias Jopp (Hrsg.): Handbuch zur deutschen Europapolitik, Baden-Baden 2021, S. 287-300.

9 Florian Eder: Brussels Playbook: Get used to Brussels - Bazooka tales - Euro bonds or euro bandage?, in: POLITICO, 26. März 2020.

10 Becker: Nach dem EU-Gipfel: Historische Integrationsschritte unter Zeitdruck, 2020. 
den European Green Deal sowie die Digitalisierung zur Verfügung stellen und durch Schuldenaufnahme an den Kapitalmärkten finanziert werden sollte. ${ }^{11}$

Am 8. Juli stimmte Merkel sodann in ihrer Rede zum Programm der deutschen Ratspräsidentschaft das Europäische Parlament auf die kommenden Entscheidungen auf der geplanten Sondertagung des Europäischen Rates am 17. Juli 2020 ein. Sie betonte, dass „Europa geeint und gestärkt“ aus der Krise hervorgehen müsse, und forderte eine „außergewöhnliche und einmalige Kraftanstrengung“von 500 Milliarden Euro angesichts des tiefen wirtschaftlichen Einbruchs durch die Pandemie. ${ }^{12}$

Die deutsch-französische Initiative stärkte der Europäischen Kommission den Rücken, die allerdings noch einen Schritt weiter ging und schon am 27. Mai 2020 einen Aufbaufonds im Rahmen ihres MFR-Vorschlags von 750 Milliarden Euro vorsah. Dass sich in diese Richtung unter deutscher Ratspräsidentschaft tatsächlich ein Konsens herausbilden könnte, zeigte sich auch an Merkels Zugehen auf Italien, indem sie sich wenige Tage vor dem Juli-Gipfel des Europäichen Rates mit dem italienischen Ministerpräsidenten Giuseppe Conte traf, auf der gemeinsamen Pressekonferenz den Kommissionsvorschlag lobte und es für eine "gute Idee“ der Kommission hielt, die Vergabe von Mitteln aus dem europäischen Aufbaufonds „an das Europäische Semester zu koppeln“, Vereinbarungen mit den einzelnen Mitgliedstaaten zu schließen und für Darlehen spätere Rückzahlungen vorzusehen. ${ }^{13}$ Damit waren die Weichen für den Sondergipfel gestellt.

Es wurde dennoch hart zwischen den Regierungschefs der Mitgliedstaaten gerungen. Die Höhe des Kommissionsvorschlags für den MFR von 1,14 Prozent des Bruttoinlandsprodukts (BIP) der EU wurde auf 1,06 Prozent des EU-BIP (1,074 Billionen Euro) gekürzt. Auch die von der Kommission vorgeschlagene Aufteilung von NGEU in 500 Milliarden Euro an Direktzuschüssen und 250 Milliarden Euro an zurückzuzahlenden Darlehen übernahm der Europäischen Rat nicht. Stattdessen einigte er sich auf 390 Milliarden Euro nicht rückzahlbare Zuschüsse und 360 Milliarden Euro Darlehen. ${ }^{14}$ Diese Haushaltseckwerte waren vor allem auf die Durchsetzungskraft der "Sparsamen Vier“ (Österreich, die Niederlande, Dänemark, Schweden $)^{15}$ zurückzuführen, die als Nettozahler im Hinblick auf Kostendämpfung beim regulären EU-Haushalt ähnliche Positionen vertraten wie Deutschland. ${ }^{16}$ Zudem hatten die "Sparsamen Vier“ die Beitragsrabatte für sich und im Übrigen

11 Presse- und Informationsamt der Bundesregierung: Deutsch-französische Initiative zur wirtschaftlichen Erholung Europas nach der Coronakrise, Pressemitteilung 173, 18. Mai 2020.

12 Presse- und Informationsamt der Bundesregierung: Rede von Bundeskanzlerin Merkel zur deutschen EURatspräsidentschaft 2020 vor dem Europäischen Parlament am 8. Juli 2020 in Brüssel, abrufbar unter: https:// www.bundesregierung.de/breg-de/aktuelles/rede-von-bundeskanzlerin-merkel-zur-deutschen-eu-ratspraeside ntschaft-2020-vor-dem-europaeischen-parlament-am-8-juli-2020-in-bruessel-1767368 (letzter Zugriff: 4.3.2021). Schon zuvor hatte Merkel auch den Bundestag auf die Notwendigkeit der Ausgabe von EU-Anleihen unter Wahrung der Haushaltsrechte der nationalen Parlamente durch die Integration des Aufbaufonds in den MFR eingestimmt; siehe Die Bundeskanzlerin: Regierungserklärung von Bundeskanzlerin Merkel in Berlin vor dem Deutschen Bundestag, 18. Juni 2020, abrufbar unter: https://www.bundeskanzlerin.de/bkin-de/aktuelles/r egierungserklaerung-von-bundeskanzlerin-merkel-1762594 (letzter Zugriff: 2.3.2021).

13 Die Bundeskanzlerin: Pressekonferenz von Bundeskanzlerin Merkel und Ministerpräsident Conte am 13. Juli 2020 in Meseberg, abrufbar unter: https://www.bundeskanzlerin.de/bkin-de/aktuelles/pressekonferenz-von-bu ndeskanzlerin-merkel-und-ministerpraesident-conte-am-13-juli-2020-1768530 (letzter Zugriff: 2.3.2021).

14 Europäischer Rat: Außerordentliche Tagung des Europäischen Rates (17., 18., 19., 20. und 21. Juli 2020) Schlussfolgerungen, EUCO 10/20.

15 Bisweilen schloss sich diesen auch Finnland an.

16 Julian Plottka/Lea Stallbaum/Zekije Bajrami: Die europapolitischen Positionen der deutschen Bundesregierung, in: Zekije Bajrami/Claudia Crawford/Livia Puglisi/Lea Stallbaum/Michael Stellwag/Julian Plottka: Poten- 
auch Deutschland durchgesetzt. Die Kommission wurde ermächtigt, zeitlich bis Ende 2026 befristet, am Kapitalmarkt Geld bis zur Höhe von 750 Milliarden Euro aufzunehmen. ${ }^{17}$ Die Schulden jenseits der rückzahlbaren Darlehen sollen durch Anhebung der Eigenmittelobergrenze um 0,6 Prozent sowie durch neue Einnahmequellen, für die ab Januar 2021 eine Kunststoffabfallabgabe und voraussichtlich ab Anfang 2023 eine $\mathrm{CO}_{2}$-Steuer und eine Digitalabgabe vorgesehen sind, innerhalb von 38 Jahren bis 2058 getilgt werden. In der Diskussion für die Einnahmenerhöhung sind zudem ein teureres Emissionshandelssystem und eine Finanztransaktionssteuer, deren Einführung von der Bundesregierung schon seit Jahren unterstützt wird. ${ }^{18}$

Allen EU-Mitgliedstaaten war offensichtlich während des langen Europäischen Rates vom 17. bis zum 21. Juli bewusst, dass es nicht nur um das schwierige Schnüren eines für die nächsten Jahre tragfähigen Finanzpakets ging, sondern dass auch die Handlungsfähigkeit der EU auf dem Spiel stand. Der auf dem Gipfel erzielte Kompromiss bediente dann viele verschiedene Interessen: den geglückten Schulterschluss zwischen Deutschland und Frankreich, die Einsparungen beim regulären MFR auf Wunsch der „Sparsamen Vier“ (was sich mit deutschen Interessen deckte) einschließlich einer Strukturverschiebung zwischen Darlehen und Zuwendungen innerhalb von NGEU sowie den erfolgreichen Kampf Italiens und der übrigen südlichen Mitgliedstaaten um eine großzügige EU-Unterstützung in der Krise. ${ }^{19}$

\section{Rechtsstaatlichkeit}

Merkel hatte neben dem Zusammenhalt der EU-27 die Rechtsstaatlichkeit als eines der zwei Leitprinzipien zu Beginn der deutschen Ratspräsidentschaft genannt. ${ }^{20}$ Da sich Art. 7 des Vertrags über die Europäische Union (EUV) bei den rechtsstaatlichen Verwerfungen in Polen und Ungarn, wo zusehends die Unabhängigkeit der Justiz untergraben und die Medien- und Pressefreiheit eingeschränkt wurde, als wenig wirksam erwiesen hatte, gab es schon seit Längerem die Idee, die Vergabe von EU-Mitteln an die Befolgung des Wertekanons in Art. 2 EUV zu binden. Die Kommission hatte hierzu bereits im Mai 2018 eine entsprechende Verordnung vorgeschlagen, ${ }^{21}$ die Deutschland und Frankreich sowie andere Mitgliedstaaten wie die Niederlande oder Schweden unterstützten. Bedeutsam wurde die Sache aber erst durch die Verknüpfung mit den Haushaltsverhandlungen über die nächste 7-Jahresphase (2021-2027). ${ }^{22}$ Diese Verknüpfung erwies sich als Fluch und Segen gleicher-

ziale deutsch-österreichischer Zusammenarbeit. Hintergrundpapier zum Wiener Dialog 2020, November 2020, S. 20ff. sowie die Kurzfassung von Zekije Bajrami/Livia Puglisi/Lea Stallbaum/Michael Stellwag/Julian Plottka: Potenziale einer österreichisch-deutschen Zusammenarbeit in der intergouvernementalen Europapolitik, in: integration 3/2020, S. 186-205.

17 Europäischer Rat: Schlussfolgerungen, Juli 2020, EUCO 10/20, S. 3.

18 Beatriz Rios/Sam Morgan: EU-Rat einigt sich: Einschnitte im Haushalt, wenig Drängen auf Rechtsstaatlichkeit, in: EURACTIV, 21. Juli 2020; Europäischer Rat: Schlussfolgerungen, Juli 2020, EUCO 10/20.

19 Matthias Rüb/Hans-Christian Rössler: „Ein schöner Sieg für Italien“ (Südeuropäer loben EU-Paket), in: Frankfurter Allgemeine Zeitung, 21. Juli 2020.

20 Presse- und Informationsamt der Bundesregierung: Rede von Bundeskanzlerin Merkel zur deutschen EURatspräsidentschaft 2020, 2020.

21 Europäische Kommission: Vorschlag für eine Verordnung des Europäischen Parlaments und des Rates über den Schutz des Haushalts der Union im Falle von generellen Mängeln in Bezug auf das Rechtsstaatsprinzip in den Mitgliedstaaten, $\operatorname{COM}(2018) 324$ final.

22 Siehe hierzu auch Ellen Bos/Kristina Kurze: Zur Einführung einer Rechtsstaatskonditionalität in der Europäischen Union: die Corona-Krise als „Window of Opportunity“, in diesem Heft, S. 23-39. 
maßen, indem sie beiden Seiten Erpressungsmöglichkeiten an die Hand gab: einmal der EU-Mehrheit gegenüber den die Grundwerte der EU systematisch verletzenden Mitgliedstaaten, die zudem noch wie Polen und Ungarn zu den größten Nettoempfängern der EU gehören, und zum anderen den beiden letztgenannten gegenüber der Mehrheit der EUMitgliedstaaten, die dringend auf die einstimmige Verabschiedung des Haushalts und des neuen Aufbaufonds wartete, wenn ab Januar 2021 die EU-Mittel fließen sollten. Zwar hätte man den NGEU-Fonds notfalls auch außerhalb des EU-Rahmens ohne Berücksichtigung der Problemstaaten intergouvernemental beschließen können, aber das entsprach vor allem nicht den Interessen der deutschen Ratspräsidentschaft. Merkel hatte bereits 2017 und 2018 bei den Vorstößen Macrons für die Einrichtung eines separaten und intergouvernementalen Haushalts für die Eurozone klargestellt, dass ein antizyklisches Instrument nur mit dem ordentlichen Haushaltsverfahren unter Beteiligung des Europäischen Parlaments geschaffen werden könne, ${ }^{23}$ was ja dann auch für den ursprünglichen MFR-Haushaltsplan in bescheidener Höhe eingeplant war. ${ }^{24}$

Die Europäische Kommission hatte in ihrem Verordnungsvorschlag noch vom Schutz der Haushaltsinteressen der Union „im Falle von generellen Mängeln in Bezug auf das Rechtsstaatsprinzip “25 gesprochen und ein umgekehrtes Mehrheitsprinzip vorgeschlagen, sodass ein betroffener Staat Sanktionen nur abwehren konnte, wenn er eine qualifizierte Mehrheit im Rat gegen den Vorschlag der Kommission organisieren konnte. ${ }^{26}$ Der Europäische Rat reduzierte dann aber im Juli 2020 den Rechtsstaatsmechanismus viel stärker auf den „Schutz der finanziellen Interessen“ der EU und bestand statt eines Sanktionsautomatismus auf einem Beschluss des Rates mit qualifizierter Mehrheit über die Kürzung von EU-Fördergeldern eines Mitgliedstaats. ${ }^{27}$ Der Rat griff dies in seinen Standpunkten vom 30. September und 14. Dezember 2020 auf, sprach ebenfalls nur noch von einer Konditionalität zum Schutz des Unionsbudgets und setzte bis zum Ende der deutschen Ratspräsidentschaft eine qualifizierte Mehrheitsentscheidung des Rates über Sanktionen durch. ${ }^{28}$ Ferner legte er einschränkend fest, dass die zu ergreifenden Maßnahmen nur proportional zu den Auswirkungen der Verstöße gegen Rechtsstaatlichkeitsprinzipien auf das EU-Budget und die finanziellen Interessen der Union sein dürfen. ${ }^{29}$

Die Verhandlungen mit dem Europäischen Parlament, das sich in seiner Position am Kommissionsvorschlag orientierte, gestalteten sich für die deutsche Ratspräsidentschaft

23 Die Bundesregierung: Erklärung von Meseberg. Das Versprechen Europas für Sicherheit und Wohlstand erneuern, Pressemitteilung 214, 19. Juni 2018.

24 Macron hatte von 100 oder 200 Milliarden Euro gesprochen, während in den MFR-Planungen nur noch 25 Milliarden Euro auftauchten, von denen noch ca. 8 Milliarden Euro an Heranführungshilfen als Anreiz für Mitgliedstaaten, die noch nicht dem Euroraum angehören, vorgesehen waren.

25 Europäische Kommission: Vorschlag für eine Verordnung über den Schutz des Haushalts, 2018.

26 Ebenda, Art. 5 Abs. 6 und 7.

27 Europäischer Rat: Schlussfolgerungen, Juli 2020, EUCO 10/20, Anlage, S. 15-16, Ziff. 22-23.

$28 \mathrm{Zu}$ den Standpunkten des Rates siehe Rat der Europäischen Union: Multiannual Financial Framework (MFF) 2021-2027 and Recovery Package - Regulation of the European Parliament and of the Council on a general regime of conditionality for the protection of the Union budget, Interinstitutional File: 2028/0136(COD), Dok. 11322/20; und Standpunkt (EU) Nr. 16/2020 des Rates in Erster Lesung im Hinblick auf den Erlass einer Verordnung des Europäischen Parlaments und des Rates über eine allgemeine Konditionalitätsregel zum Schutz des Haushalts der Union, vom Rat am 14. Dezember 2020 angenommen, in: Amtsblatt der EU, Nr. C 441 vom 18. Dezember 2020, S. 1-10.

29 Ebenda, Art. 5 Abs. 3; siehe auch schon zuvor den Standpunkt des Rates in Rat der Europäischen Union: Regulation on a general regime of conditionality, Dok. 11322/20, Annex, 2020, Art. 4 Abs. 3. 
entsprechend schwierig; eine Einigung gelang erst am 5. November 2020. ${ }^{30}$ Die deutsche Ratspräsidentschaft, die durchaus mit den Positionen des Parlaments sympathisierte, musste in ihrer Vorsitz-Funktion als „ehrlicher Makler“ zwischen Rat und Parlament verhandeln und hatte auch den am Ende erforderlichen Einstimmigkeitszwang für die Verabschiedung des Gesamtpakets im Auge (obwohl für die Verabschiedung der Verordnung selbst nur eine qualifizierte Mehrheit erforderlich war). Das Parlament konnte sich zwar in vielen Erwägungsgründen des Verordnungstextes durchsetzen, hatte im Kern aber wenig ändern können, denn es blieb bei der Letztentscheidung des Rates mit qualifizierter Mehrheit, sollte die Kommission eine Suspendierung von Zahlungen oder die Kürzung von EUMitteln vorschlagen. Auch gelang es dem Europäischen Parlament nicht, den „Bremsmechanismus“ abzuschaffen. ${ }^{31}$ Hierbei geht es um die Möglichkeit des betroffenen Mitgliedstaats, den Europäischen Rat als Konsens- oder Einstimmigkeitsgremium mit einer Diskussion über seinen Fall - und dies mit aufschiebender Wirkung - zu befassen, wodurch dieser Mitgliedstaat zumindest die Möglichkeit einer zeitlichen Verzögerung hat.

Die deutsche Ratspräsidentschaft sah in der mühsam erreichten politischen Einigung mit dem Parlament am 5. November einen Durchbruch zum Schutz des EU-Budgets gegen Missbrauch von EU-Geldern. ${ }^{32}$ Das Parlament zeigte sich ebenfalls zufrieden, weil das neue Gesetz nicht nur bei „Korruption oder Betrug“, sondern auch bei „systemischen Verstößen gegen [...] EU-Grundwerte“ angewandt werden könne. ${ }^{33}$ Es war bei den harten Positionen des Rates offensichtlich auch deshalb kompromissbereit, weil eine Einigung über den MFR und den NGEU-Aufbaufonds noch ausstand und es in den Verhandlungen mit dem deutschen Ständigen Vertreter Clauß um eine Erhöhung bestimmter Budgetpositionen rang. ${ }^{34}$ Hierüber konnte dann auch erst fünf Tage nach der politischen Annahme des Rechtsstaatsmechanismus, am 10. November 2020, eine Einigung erzielt werden, wobei die Mehrheit des Europäischen Parlaments letztlich das Finanzpaket von 1,8 Billionen Euro nicht durch Maximalforderungen blockieren wollte. Diese Sequenzialisierung (erst Rechtsstaatsmechanismus, dann Budget) hatte der deutschen Ratspräsidentschaft in den Verhandlungen mit dem Parlament einen kleinen Vorteil verschafft, um in beiden Bereichen eine aus Ratssicht realistische Einigung zu erzielen.

Das Parlament zeigte sich am Ende dennoch zufrieden, im neuen MFR 2021-2027 ca. 16 Milliarden Euro mehr für EU-Programme wie EU4Health, Erasmus, das Forschungsrah-

30 Rat der Europäischen Union: Konditionalität für den Haushalt: Verhandlungsführer des Ratsvorsitzes und des Parlaments erzielen vorläufige Einigung, Pressemitteilung 750/20, 5. November 2020.

31 Europäische Kommission: Mitteilung der Kommission an das Europäische Parlament gemäß Artikel 294 Absatz 6 des Vertrags über die Arbeitsweise der Europäischen Union betreffend den Standpunkt des Rates im Hinblick auf den Erlass einer Verordnung des Europäischen Parlaments und des Rates über eine allgemeine Konditionalitätsregelung zum Schutz des Haushalts der Union, COM(2020) 843 final; sowie Europäisches Parlament: Entschließungsantrag zum Mehrjährigen Finanzrahmen 2021-2027, der interinstitutionellen Vereinbarung, dem EU-Aufbauinstrument und der Verordnung über die Rechtsstaatlichkeit, 14. Dezember 2020, B9-0428/2020; Rat der Europäischen Union: Standpunkt Nr. 16/2020, Abs. 26.

32 So der deutsche Ständige Vertreter Clauß nach erzielter Einigung mit dem Europäischen Parlament in Rat der Europäischen Union: Konditionalität für den Haushalt, 2020.

33 Europäisches Parlament: Rechtsstaatlichkeit und EU-Haushalt: Abgeordnete und Rat erzielen Kompromiss, Pressemitteilung, 5. November 2020.

34 Siehe hierzu schon die Forderungen des Europäischen Parlaments direkt nach dem Sondergipfel des Europäischen Rates im Juli 2020 in Europäisches Parlament: Entschließungsantrag zu den Schlussfolgerungen der außerordentlichen Tagung des Europäischen Rates vom 17.-21. Juli 2020 (2020/2732(RSP)), 23. Juli 2020, P9_TA(2020)0206. 
menprogramm Horizon Europe, den EU-Grenzschutz, die humanitäre Hilfe, die Unterstützung von Staaten der Europäischen Nachbarschaftspolitik (ENP) und die Entwicklungszusammenarbeit durchgesetzt sowie einen rechtsverbindlichen Fahrplan zur Einführung neuer Finanzmittel für die EU und rechtlich verbindliche Klimaschutzausgaben von 30 Prozent im MFR sowie bei Mitteln aus dem Aufbaufonds erstritten zu haben. ${ }^{35}$

Trotz der erzielten Einigung zwischen Rat und Parlament Anfang November 2020 war das weitere Verfahren blockiert, da Polen und Ungarn den Rechtsstaatsmechanismus in der bestehenden Form der Verordnung nicht akzeptierten und Änderungen verlangten, bevor sie dem Finanzpaket der EU zustimmen würden. Dies führte zu einem Anwachsen der Spannungen in der EU und verkomplizierte die Lage gerade auch für die deutsche Ratspräsidentschaft erheblich: Der MFR- und der Eigenmittelbeschluss mussten einstimmig im Rat und der MFR zuvor auch im Europäischen Rat verabschiedet werden, wobei der Eigenmittelbeschluss zur Finanzierung der Ausgaben zusätzlich noch eine Ratifizierung durch die nationalen Parlamente erforderte.

Es gelang erst nach zähen Verhandlungen zwischen der Ratspräsidentschaft und den beiden Ländern, den Weg für einen Kompromiss zu ebnen, der im Europäischen Rat dann noch festgeklopft werden musste. Dieser Kompromiss bestand in einer umfangreichen Erklärung des Europäischen Rates, die freilich den Rechtstext der Verordnung unangetastet ließ. Diese gilt so wie verabschiedet trotz aller Blockadedrohungen Ungarns und Polens. Der Europäische Rat konzedierte lediglich eine aufschiebende Wirkung im Falle einer Nichtigkeitsklage vor dem Gerichtshof der Europäischen Union (EuGH), die grundsätzlich jedem Mitgliedstaat zusteht. In einem solchen Fall soll die Kommission ihre Leitlinien für die Anwendung der Verordnung erst nach einem (abschlägigen) Urteil des EuGH und in dessen Licht erstellen und bis dahin keine Maßnahmen vorschlagen. ${ }^{36}$ Alle anderen Erklärungen in den Schlussfolgerungen des Europäischen Rates zur Sache befinden sich schon im Text des Verordnungsvorschlags. Erst diese Einigung im Europäischen Rat ermöglichte es Rat und Parlament, das Gesamtpaket von MFR-Verordnung und Eigenmittelbeschluss nach wochenlanger Verzögerung anzunehmen. Der Text des Europäischen Rates hatte insofern noch ein besonderes Gewicht, als er nicht nur von Merkel als Ratspräsidentin, sondern auch von der Kommissionspräsidentin direkt mit ausgehandelt worden und trotz heftiger Kritik des Europäischen Parlaments an der aufschiebenden Wirkung auch für die Kommission verbindlich war. ${ }^{37}$

Mehr war aufgrund der Gesamtsituation nicht möglich, wobei ein weiterer Grund für die Entschärfung des Rechtsstaatsmechanismus durch Abschaffung des Kürzungsautomatismus (umgekehrte qualifizierte Mehrheit im Rat) sicherlich auch darin bestand, dass die

35 Europäisches Parlament: Entschließung des Europäischen Parlaments vom 17. Dezember 2020 zum Mehrjährigen Finanzrahmen 2021-2027, der interinstitutionellen Vereinbarung, dem EU-Aufbauinstrument und der Verordnung über die Rechtsstaatlichkeit (2020/2923(RSP), 17. Dezember 2020, P9_TA(2020)0360.

36 Europäischer Rat: Tagung des Europäischen Rates (10. und 11. Dezember 2020) - Schlussfolgerungen, EUCO 22/20, Ziff. 2c) sowie 2e) und 2f).

37 Das Europäische Parlament hat seine Kritik an der Erklärung des Europäischen Rates und am gesamten Verfahren sehr deutlich in seinen Erwägungsgründen dargestellt in: Entschließung zum Mehrjährigen Finanzrahmen 2021-2027, der interinstitutionellen Vereinbarung, dem EU-Aufbauinstrument und der Verordnung über die Rechtsstaatlichkeit (2020/2923 (RSP), insbesondere Erwägung E4 und E9. 
Mitgliedstaaten der Kommission beim Rechtsstaatsmechanismus offensichtlich keine zu starke Rolle in einem hochsensiblen innerstaatlichen Bereich zukommen lassen wollten. ${ }^{38}$

Dass eine Verknüpfung von generellen Rechtsstaatsdefiziten und Kürzungen der Budgetzuweisungen bei einem Mitgliedstaat, der die Grundwerte der EU systematisch verletzt, nicht gelang, ist zweifellos ein Makel, der durch die Fokussierung auf Unregelmäßigkeiten in der Mittelverwendung nicht gänzlich beseitigt werden kann. Die Auffassung, dass die EU „ihre Autokraten davonkommen“ lasse oder der Rechtsstaat der eigentliche „Verlierer“ sei ${ }^{39}$ lässt sich aber so nicht aufrechterhalten. Es ist vielmehr das Verdienst der deutschen Ratspräsidentschaft, wesentliche Kernelemente der Rechtsstaatsverordnung erhalten und ein „Stoppschild für Autoritäre“ aufgestellt zu haben. ${ }^{40}$ Gerade in autokratisch regierten Ländern wie Polen und insbesondere Ungarn, die sich der Europäischen Staatsanwaltschaft (EUStA) zur Bekämpfung von Korruption im Zusammenhang mit EU-Geldern nicht angeschlossen haben, ${ }^{41}$ werden teilweise EU-Mittel in klientelistischer Weise verwendet, um die Machtbasis der Regierungsparteien zu erweitern. ${ }^{42}$ Die Verordnung stellt nunmehr ein zusätzliches Instrument dar, welches es in dieser Form bislang nicht gegeben hat und zudem nicht nur für Polen und Ungarn geschaffen wurde, sondern auch in anderen Fällen bei Korruption oder Vetternwirtschaft, etwa mit Blick auf Bulgarien, Rumänien, Malta und andere, anwendbar ist. Das qualifizierte Mehrheitserfordernis im Rat ist hierbei eine Hürde, lässt aber doch auf eine solide Mehrheit der liberalen Demokratien im Rat hoffen. ${ }^{43}$

\section{Koordination der Gesundheitspolitik und gemeinsame Impfstrategie}

Was die Bekämpfung gesundheitsbezogener Auswirkungen betrifft, so gewähren die Verträge der EU nur begrenzte Zuständigkeiten. Diese beziehen sich im Wesentlichen auf die Gesundheitspolitik als Querschnittsaufgabe, ergänzen die Gesundheitspolitiken der Mitgliedstaaten und fördern deren Kooperation in Gesundheitsfragen (Art. 168 des Vertrags über die Arbeitsweise der Europäischen Union, AEUV). Angesichts der COVID-19Pandemie hat die Europäische Kommission die Gesamtkoordinierung der Maßnahmen inklusive der Vorgehensweise bei der Beschränkung der Freizügigkeit übernommen. ${ }^{44} \mathrm{Da}$ -

38 Raphael Bossong: Rechtsstaatlichkeit in der EU: Die Debatte über eine Einschränkung von Finanzzahlungen und ungelöste Grundsatzkonflikte, Stiftung Wissenschaft und Politik: SWP-Aktuell 72/2020.

39 Markus Becker: Und der Verlierer ist... der Rechtsstaat, in: Der Spiegel, 10. Dezember 2020; in ähnlicher Richtung argumentierte er schon nach dem Juli-Gipfel, siehe Markus Becker: Die EU lässt ihre Autokraten davonkommen, in: Der Spiegel, 21. Juli 2020.

40 Matthias Krupa: Ein Stoppschild für die Autoritären, in: Zeit Online, 11. Dezember 2020; Thomas Gutschker: Haben Polen und Ungarn nichts mehr zu befürchten?, in: Frankfurter Allgemeine Zeitung, 11. Dezember 2020.

41 Außer ihnen sind es noch Irland und Dänemark wegen ihres Opt-outs vom Raum der Freiheit, der Sicherheit und des Rechts, die nicht teilnehmen, sowie Schweden, das aber einen Anschluss erwägt. Alle anderen 22 Mitgliedstaaten nehmen an der EUStA teil.

42 Europäische Kommission: Bericht über die Rechtsstaatlichkeit 2020. Die Lage der Rechtsstaatlichkeit in der Europäischen Union, $\operatorname{COM}(2020) 580$ final; sowie insbesondere Europäische Kommission: Bericht über die Rechtsstaatlichkeit 2020. Länderkapitel zur Lage der Rechtsstaatlichkeit in Polen, SWD(2020) 320 final, S. 1115; und Europäische Kommission: Bericht über die Rechtsstaatlichkeit 2020. Länderkapitel zur Lage der Rechtsstaatlichkeit in Ungarn, SWD(2020) 316 final, S. 11-14.

43 Eine Blockademinderheit würde mindestens vier Staaten und 156 Millionen BürgerInnen erfordern. Polen und Ungarn verfügen zusammen aber nur über knapp 50 Millionen EU-BürgerInnen. Es dürfte ihnen deshalb schwer fallen, einen qualifizierten Mehrheitsbeschluss im Rat auszubremsen.

44 Europäischer Rat: Tagung des Europäischen Rates (15. und 16. Oktober 2020) - Schlussfolgerungen, EUCO $15 / 20$. 
rüber hinaus haben sich die Mitgliedstaaten auch aufgrund des starken Fürspruchs der deutschen Ratspräsidentschaft im Kampf gegen COVID-19 zusammengeschlossen und eine gemeinsame Impfstoffstrategie entwickelt. Diese beinhaltet, dass die Europäische Arzneimittel-Agentur mit der Bewertung und Empfehlung der Anwendung der COVID-19Impfungen für alle 27 EU-Mitgliedstaaten beauftragt wurde. Darüber hinaus hat die Kommission eine gemeinsame Impfstrategie entwickelt, die mit Zustimmung der Mitgliedstaaten erstmalig ihre direkten Verhandlungen mit mehreren Pharmaunternehmen umfasst, um genügend Impfdosen für alle europäischen BürgerInnen zu sichern. ${ }^{45}$ Eine solche Stärkung der Rolle der Kommisssion in der Gesundheitspolitik ist präzedenzlos und ein weiteres Beispiel für selektive Föderalisierung.

\section{Externe Herausforderungen für die EU: Außenpolitik und Außenwirtschaftspolitik}

In den Zeitraum der deutschen Ratspräsidentschaft fielen zahlreiche außenpolitische Herausforderungen in der Nachbarschaft und auf der Bühne der Weltpolitik, auf die reagiert werden musste. Neben den hier beleuchteten Beziehungen zum Vereinigten Königreich, der Lage im östlichen Mittelmeer, den Protesten nach den Präsidentschaftswahlen in Belarus und den EU-Russland-Beziehungen nach dem Giftanschlag auf den russischen Oppositionellen Alexej Nawalny ging es in der Nachbarschaft auch um die Aushandlung eines Waffenstillstands nach dem Wiederaufflammen des Krieges zwischen Armenien und Aserbaidschan um die Region Bergkarabach, bei der die EU nur eine geringe Rolle spielte, wie von manchen Abgeordneten des Europäischen Parlaments bemängelt wurde. ${ }^{46}$ Weltpolitische Entwicklungen, die im Fokus der deutschen Ratspräsidentschaft standen, waren zum einen die transatlantischen Beziehungen und zum anderen die Entwicklungen der EU-China-Beziehungen.

\section{Die Beziehungen zum Vereinigten Königreich nach dem Brexit}

Mit einer nicht zu überbietenden Dramatik ${ }^{47}$ kam es unter der deutschen Ratspräsidentschaft zum Showdown in den Verhandlungen über die Gestaltung der künftigen Beziehungen zwischen der EU und dem Vereinigten Königreich, indem man sich ausgerechnet am Heiligen Abend, dem 24. Dezember 2020, auf ein Handels- und Kooperationsabkommen einigte, was von deutscher Seite wegen der traditionell intensiven Handelsbeziehungen zu dem Land mit großer Erleichterung aufgenommen wurde. ${ }^{48}$ Das Abkommen entspricht nicht ganz dem EU-Kanada-Abkommen und bietet Freihandel bei Waren und Dienstleis-

45 Ebenda.

46 Barbara Wessel: EU machtlos in Berg-Karabach, in: Deutsche Welle, 7. Oktober 2020; Rat der Europäischen Union: Bergkarabach: Erklärung des Hohen Vertreters im Namen der Europäischen Union, Pressemitteilung 793/20, 19. November 2020; Statement von Viola von Cramon-Taubadel (MdEP): „Ernsthafte Bemühungen zur Lösung der Krise in Berg-Karabach waren seitens der deutschen Ratspräsidentschaft nicht erkennbar. Die Passivität der Ratspräsidentschaft und der EU hat unter den Konfliktparteien zu großer Unzufriedenheit geführt.“, in: Europagruppe Grüne: Deutsche EU-Ratspäsidentschaft: Eine Zwischenbilanz der Europagruppe Grüne, 13. November 2020, abrufbar unter: https://sven-giegold.de/deutsche-eu-ratspraesidentschaft-gruenezwischenbilanz/ (letzter Zugriff: 28.1.2021).

47 Vgl. auch zur Dramaturgie des gesamten Brexitprozesses: Nicolai von Ondarza: Bekanntes Brexit-Theater, neue Rahmenbedingungen, Stiftung Wissenschaft und Politik: Kurz gesagt, 22. September 2020.

48 Frankfurter Allgemeine Zeitung: Brexit-Handelspakt: Merkel nennt Einigung historisch, 25. Dezember 2020. 
tungen. ${ }^{49}$ Es klammert aber Finanzdienstleistungen aus, bei denen sich das Vereinigte Königreich nicht auf das EU-Regelungsniveau einlassen wollte, obwohl gerade dieser Bereich den Briten aufgrund komparativer Vorteile besonders wichtig war. Auch musste Boris Johnson das höchst umstrittene völkerrechtswidrige Binnenmarktgesetz, das Teile der Nordirlandregelung im Protokoll zum Austrittsabkommen mit der EU aushebeln wollte, wesentlich entschärfen. Er scheiterte an der ablehnenden Haltung des House of Lords und vor allem an der Einmischung des designierten amerikanischen Präsidenten Joe Biden, der auch wegen seiner irischen Familienwurzeln auf die Einhaltung des Karfreitagsabkommens und des Austrittsabkommens mit der EU drängte. Das schränkte den Handlungsspielraum Johnsons ein und machte ihn kompromissbereiter. ${ }^{50}$

Deutschland hatte von Anfang an den Brexit intensiv begleitet, denn etwa 6 bis 8 Prozent der jährlichen Exporte des Landes gingen traditionell in das Vereinigte Königreich, auch wenn sie mittlerweile rückläufig sind. ${ }^{51} \mathrm{Da}$ das Vereinigte Königreich dennoch ein wichtiger Markt bleibt, ${ }^{52}$ wollte man aufseiten der deutschen Wirtschaft - nachdem sich die Hoffnungen auf einen Regierungswechsel oder ein zweites, den Brexit revidierendes Referendum durch den Wahlsieg Johnsons im Dezember 2019 zerschlagen hatten wenigstens ein zoll- und kontingentfreies Handelsabkommen, um einen No-Deal-Brexit abzuwenden. Dies war auch für die Bundesregierung maßgeblich, deren Interessen nach der Hängepartie über den Austrittsvertrag und das Austrittsdatum in die im Februar 2020 erlassenen Richtlinien des Rates für die Verhandlungen mit dem Vereinigten Königreich umfänglich eingeflossen waren. ${ }^{53}$

Während der Ratspräsidentschaft galten für die Bundesregierung wie im gesamten Brexit-Prozess zuvorderst zwei Leitprinzipien: Der Zusammenhalt der EU-27 musste gestärkt werden und der Binnenmarkt mit seinen vier Freiheiten erhalten bleiben, was Sonderzugangsrechte zum EU-Binnenmarkt für das Vereinigte Königreich etwa bei Finanzdienstleistungen ausschloss. Es ging deshalb in den Verhandlungen aus deutscher Sicht um Freihandel zu fairen Wettbewerbsbedingungen mit Beihilfekontrollen, Reziprozität und Erleichterung von Grenzformalitäten. Die Fischereipolitik, über die bis zuletzt heftig gestritten wurde, war kein besonders wichtiges Thema für Deutschland. Aber für Frankreich stand es oben auf der Agenda, was sich auch auf die deutsche Haltung auswirkte, denn bei allen Verhandlungen mit dem Vereinigten Königreich kam es der Bundesregierung seit 2016 immer auf den deutsch-französischen Schulterschluss an. Diese Prinzipien und Zusammenhänge waren von den Tory-geführten Regierungen nie verstanden worden,

49 Anna Isaac: 10 key details in the UK-EU trade deal, in: POLITICO, 5. Januar 2021; Handels- und Kooperationsabkommen zwischen der Europäischen Union und der Europäischen Atomgemeinschaft einerseits und dem Vereinigten Königreich Großbritannien und Nordirland andererseits, in: Amtsblatt der EU, Nr. L 444 vom 31. Dezember 2020, S. 14-1462.

50 Jochen Buchsteiner: Warum Johnson wenig Spielraum hat, in: Frankfurter Allgemeine Zeitung, 8. Dezember 2020; Nicolai von Ondarza: Boris Johnson unter Druck: Was bedeutet der Wahlsieg Bidens für den Brexit?, Stiftung Wissenschaft und Politik: Kurz gesagt, 18. November 2020.

51 Zeit Online: Deutlich weniger deutsche Exporte nach Großbritannien, 27. Januar 2020.

52 Lisandra Flach/Feodora Teti/Lena Wiest/Margherita Atzei/Lisa Scheckenhofer: Wie abhängig sind Deutschland und die EU vom Vereinigten Königreich? Produktabhängigkeiten und die Auswirkungen des Brexit, in: ifo Schnelldienst 12/2020, S. 6-12.

53 Rat der Europäischen Union: Annex to Council Decision authorising the opening of negotiations with the United Kingdom of Great Britain and Northern Ireland for a new partnership agreement, Dok. 5870/20, ADD 1 Rev 3. 
sah man doch in Deutschland den gewichtigsten Partner in der EU, der seinen Einfluss auf die Verhandlungen geltend machen könnte, um nach dem Ausscheiden aus der EU möglichst günstige Zugänge zum EU-Markt zu erhalten. Die Bundesregierung machte aber deutlich, dass die Europäische Kommission wie in der gemeinsamen Handelspolitik üblich die Verhandlungen führte, und stand von Anfang an hinter Michel Barnier, dem Brexit-Chefunterhändler der EU. ${ }^{54}$ Das Vertrauen in sein Verhandlungsteam war groß und die Bundeskanzlerin verbreitete bis zum Abschluss der Verhandlungen Optimismus. ${ }^{55} \mathrm{Sie}$ nannte die erzielte Einigung nach viereinhalb Jahren „Springprozession“ mit den Briten mit Fug und Recht „historisch“ ${ }^{\text {“ }}{ }^{6}$ Hierbei waren die Verbindung zwischen ihr und von der Leyen sowie die der beiden zu Barnier in den letzten Verhandlungsphasen genauso hilfreich wie die zu Sabine Weyand, Leiterin der Generaldirektion Handel der Kommission, die früher zum Verhandlungsteam Barniers gehörte.

\section{Das östliche Mittelmeer}

$\mathrm{Zu}$ den außenpolitischen Problemen während der deutschen Ratspräsidentschaft gehörten die Spannungen und Konflikte im östlichen Mittelmeerraum. Die Türkei unternimmt dort schon seit zwei Jahren Explorationen und Bohrungen wegen neuerlich entdeckter und vermuteter großer Erdgasvorkommen. Dabei verletzt sie Hoheitsgewässer sowie Ausschließliche Wirtschaftszonen Griechenlands und Zyperns. ${ }^{57}$

Der Konflikt spitzte sich während der deutschen Ratspräsidentschaft zu, da Griechenland Teile seiner Flotte zur Abdrängung türkischer Bohrschiffe und Marineeinheiten ins Krisengebiet schickte. ${ }^{58}$ Dies war für die deutsche Ratspräsidentschaft eine schwierige Situation. Zwar hatte der Wissenschaftliche Dienst des Deutschen Bundestages schon im Januar 2020 hinsichtlich des maritimen Türkei-Libyen-Abkommens von einem Verstoß "gegen das völkergewohnheitsrechtliche Seerecht" gesprochen, ${ }^{59}$ doch gehörte Deutschland traditionell gegenüber den Hardlinern in der EU (Griechenland, Zypern und Frankreich) eher zu den Bremsern hinsichtlich einer härteren Gangart gegenüber der Türkei. In ihrer Rolle als Vorsitz des Rates der EU konnte die Bundesregierung jedoch nur eine Mittlerposition einnehmen, wobei sie gelegentlich die direkte Verbindung zwischen Merkel und Recep Tayyip Erdoğan nutzen konnte, um etwa im Spätherbst 2020 die Türkei zum Rückzug ihres Bohr- und Explorationsschiffes „Oruç Reis“ zu bewegen. Darüber hinaus überließ die Ratspräsidentschaft das Feld weitgehend dem Hohen Vertreter der Union für Außen- und Sicherheitspolitik Josep Borrell.

54 Die Bundeskanzlerin: Bundeskanzlerin Merkel zum Brexit: Ein tiefer Einschnitt für uns alle, 31. Januar 2020, abrufbar unter: https://www.bundeskanzlerin.de/bkin-de/aktuelles/bundeskanzlerin-merkel-zum-brexit-ein-ti efer-einschnitt-fuer-uns-alle-1717480 (letzter Zugriff: 28.1.2021).

55 Redaktionsnetzwerk Deutschland: Brexit-Handelspakt: Merkel setzt weiter auf Einigung mit Großbritannien, 30. November 2020.

56 Frankfurter Allgemeine Zeitung: Merkel nennt Einigung historisch, 2020.

57 Günter Seufert: Störenfried aus Ankara, in: Zeit Online, 28. August 2020.

58 Ozan Demircan/Gerd Höhler: Erneute Eskalation im Mittelmeer: Die Türkei fordert Griechenland und das Seerecht heraus, in: Handelsblatt, 12. August 2020.

59 Wissenschaftlicher Dienst des Deutschen Bundestags: Seevölkerrechtliche Bewertung der türkisch-libyschen Vereinbarung über die Abgrenzung ihrer maritimen Interessenssphären im östlichen Mittelmeer, 17. Januar 2020, WD 2-3000-143/19, S. 18. 
Schon im November 2019 hatte der Rat Sanktionen wegen des „anhaltenden rechtswidrigen“ Vorgehens der Türkei im östlichen Mittelmeer verhängt. ${ }^{60}$ Die Verhandlungen über das Luftverkehrsabkommen wurden ausgesetzt, die Tagungen des Assoziierungsrates mit der Türkei abgesagt und die Heranführungshilfen für 2020 gekürzt. Zudem wurden Reisebeschränkungen gegenüber natürlichen und juristischen Personen verhängt, die an den Bohrungen beteiligt oder für sie verantwortlich waren, sowie Gelder der Betreffenden eingefroren. Nach der Eskalation der Situation im August und September 2020 betonte der Europäische Rat im Oktober die „uneingeschränkte Solidarität mit Griechenland und Zypern" und forderte die Türkei auf, von Schritten abzusehen, die dem Völkerrecht und „den Interessen der EU zuwiderlaufen“ ${ }^{61}$ Er begrüßte aber auch die Absicht Griechenlands und der Türkei, gegebenenfalls Sondierungsgespräche über Festlandsockel und Ausschließliche Wirtschaftszonen führen zu wollen. Im Falle Zyperns forderte er von der Türkei, von rechtswidrigen Maßnahmen abzusehen und die Einladung Zyperns zu Gesprächen anzunehmen. Auch verwies er auf die Einhaltung einschlägiger Resolutionen der Vereinten Nationen (Resolution 550 und 789).62 Der deutschen Ratspräsidentschaft waren auch positive Anreize wichtig. So bot der Europäische Rat eine „positive politische EU-TürkeiAgenda“ an, falls die Türkei ihre „illegalen Aktivitäten“ gegenüber Griechenland und Zypern einstellt. ${ }^{63}$ Dieses Angebot an die Türkei umfasste eine Modernisierung der Zollunion, die Erleichterung der Handelsbeziehungen, bessere zwischenmenschliche Kontakte (keine Visafreiheit), einen Dialog auf hoher Ebene und die weitere Zusammenarbeit im Bereich Migration auf Basis der EU-Türkei-Erklärung von 2016. Die Schlussfolgerungen des Europäischen Rates enthielten zudem den auch von deutscher Seite besonders favorisierten Vorschlag einer „Multilateralen Konferenz" über den östlichen Mittelmeerraum, um über "Seegebiete, Sicherheit, Energie, Migration und wirtschaftliche Zusammenarbeit" zu verhandeln. ${ }^{64}$ Sollte die Türkei ihr rechtswidriges Verhalten nicht einstellen, werde die EU aber die ihr „zur Verfügung stehenden Instrumente und Optionen nutzen“65 einschließlich weiterer Sanktionen -, um ihre Interessen und die ihrer Mitgliedstaaten zu verteidigen.

Trotz dieser Kombination aus „Zuckerbrot und Peitsche“ reagierte die Türkei nur zögerlich. Sie zog zwar auch wegen der Ungewissheit über die künftige US-Außenpolitik unter Biden besagtes Explorationsschiff zurück, verschärfte aber ihre Rhetorik gegenüber der EU, deren Mitgliedstaaten und Führungspersonal, was auf dem Gipfeltreffen der Staatsund Regierungschefs im Dezember 2020 nur eine Erweiterung der Listen mit Sanktionen belegter Personen und Institutionen zur Folge hatte. Dennoch hielt der Europäische Rat

60 Beschluss (GASP) 219/1894 des Rates vom 11. November 2019 über restriktive Maßnahmen angesichts der nicht genehmigten Bohrtätigkeiten der Türkei im östlichen Mittelmeer, in: Amtsblatt der EU, Nr. L 291 vom 11. November 2019, S. 47-53.

61 Europäischer Rat: Außerordentliche Tagung des Europäischen Rates (1. und 2. Oktober 2020) - Schlussfolgerungen, EUCO 13/20, Ziff. 16-22.

62 Ebenda, Ziff. 19.

63 Ebenda, Ziff. 20; Funda Tekin: Five Years After the EU-Turkey Statement: Germany Can Contribute to Navigating a Turbulent Relationship, Institut für Europäische Politik: Berlin Perspectives 6/2020; und Funda Tekin: Fünf Jahre EU-Türkei-Erklärung und die Frage nach dem institutionellen Blickwechsel in den bilateralen Beziehungen, in: integration 4/2020, S. 295-309.

64 Europäischer Rat: Schlussfolgerungen, Oktober 2020, EUCO 13/20, Ziff. 22.

65 Ebenda, Ziff. 21. 
das Angebot einer positiven EU-Türkei-Agenda aufrecht; die Modernisierung der Zollunion wurde allerdings nicht mehr erwähnt. ${ }^{66}$

\section{Die EU-Belarus-Beziehungen nach den Präsidentschaftswahlen}

Belarus befindet sich seit den Präsidentschaftswahlen vom 9. August 2020 in einer politischen Krise. In Deutschland wie anderswo war die Erwartung bis wenige Wochen vor der Wahl, dass Alexander Lukaschenko - der autokratische Führer, der seit 1994 an der Macht ist - sich mit dem üblichen Erdrutschsieg durchsetzen würde, nachdem er die echten Herausforderer von der Kandidatur abgehalten hatte. In der Zeit vor den Wahlen kam es in Belarus jedoch zu einem überraschenden Grad an politischen Auseinandersetzungen. Zunächst durften drei Oppositionelle nicht kandidieren und wurden entweder festgenommen oder gezwungen, das Land zu verlassen. Dann bildeten drei Frauen aus ihrem Umfeld Veronika Tsepkalo (Ehefrau von Waleri Tsepkalo), Swetlana Tichanowskaja (Ehefrau von Sergej Tichanowskij) und Maria Kolesnikowa (Wiktar Babarykas Wahlkampfmanagerin) ein Wahlbündnis. Unmittelbar nachdem Lukaschenko mit 80,2 Prozent der Stimmen zum Sieger erklärt worden war, während Tichanowskaja nur 9,9 Prozent erhielt, begannen in Minsk Massenproteste, die sich bald im ganzen Land ausbreiteten. Viele DemonstrantInnen waren Polizeigewalt ausgesetzt, u.a. durch den Einsatz von Gummigeschossen und Betäubungsgranaten durch Spezialeinheiten der belarusssichen Polizei, und es gab Massenverhaftungen von DemonstrantInnen und JournalistInnen; viele berichteten, dass sie im Gefängnis gefoltert wurden. Die benachbarten EU-Länder Polen, Litauen und Lettland kritisierten neben der Ukraine den Verlauf der Wahlen. Zu den anhaltenden Protesten gehören nicht nur öffentliche Demonstrationen, sondern auch Streiks in staatlichen Unternehmen, die dezentral organisiert werden.

Deutschland bot zwar an, eine gemeinsame Position für die EU zu finden, blieb aber zurückhaltend. ${ }^{67}$ Zwei deutsche Mitglieder des Europäischen Parlaments gehörten zu jenen, die eine Erklärung veröffentlichten, in der sie das Vorgehen der belarussischen Regierung verurteilten und forderten, dass die EU mit Sanktionen drohen sollte, wenn Lukaschenko die Repressionsakte nicht beendet. ${ }^{68}$ Nach einiger Verzögerung hat die deutsche Regierung die Gewalt der belarussischen Sicherheitskräfte und die Massenverhaftungen von DemonstrantInnen und Umstehenden verurteilt. Sie hat deren Freilassung sowie die Einhaltung der Grundprinzipien freier und fairer Wahlen gefordert.

Am 19. August 2020 fand ein außerordentlicher EU-Gipfel statt, um die Krise in Belarus zu erörtern. In den Schlussfolgerungen des Präsidenten des Europäischen Rates hieß es, die EU habe die Wahlergebnisse nicht anerkannt, die Gewalt verurteilt und eine Liste mit einer beträchtlichen Anzahl von Personen erstellt, die für Gewalt, Repression und die Fälschung von Wahlergebnissen verantwortlich seien und die mit dem Einfrieren von Vermögenswer-

66 Europäischer Rat: Schlussfolgerungen, Dezember 2020, EUCO 22/20, Ziff. 31-34.

67 Katrin Böttger: After the Presidential Election in Belarus. Germany's role in formulating a united EU position defending human rights and the rule of law, Institut für Europäische Politik: Berlin Perspectives 3/2020.

68 Viola von Cramon: Gemeinsames Statement zu den belarussischen Präsidentschaftswahlen, 11. August 2020, abrufbar unter: https://violavoncramon.eu/2020/08/11/gemeinsames-statement-zu-den-belarussischen-prasid entschaftswahlen/ (letzter Zugriff: 28.1.2021). 
ten und Reiseverboten belegt werden sollten. ${ }^{69}$ Darüber hinaus sagte von der Leyen finanzielle Unterstützung in Höhe von 53 Millionen Euro für Belarus zu, das meiste davon für den Kampf gegen die COVID-19-Pandemie, 2 Millionen Euro für die Opfer der Repression und 1 Million Euro für die Zivilgesellschaft und unabhängige Medien. ${ }^{70}$ In Reaktion auf das betrügerische Vorgehen bei den Präsidentschaftswahlen verhängte der Rat der EU am 2. Oktober 2020 Sanktionen gegen Belarus. ${ }^{71}$ Ein Beschluss zu diesen Sanktionen war wochenlang von Zypern blockiert worden, da es im Gegenzug Sanktionen gegen die Türkei wegen der Erkundungsbohrungen nach Erdgas in umstrittenen Gewässern im östlichen Mittelmeer einforderte. ${ }^{72}$ Hier musste die deutsche Ratspräsidentschaft aufgrund der notwendigen Einstimmigkeit viel Vermittlungsarbeit leisten und erhielt zugleich Kritik, dass diesbezüglich nicht schneller eine Entscheidung gefunden werden konnte.

\section{Die EU-Russland-Beziehungen}

Der russische Oppositionspolitiker Nawalny war am 20. August 2020 im sibirischen Tomsk vergiftet worden. Nachdem das Flugzeug, in dem er saß, als die Symptome auftraten, in Omsk notlandete, wurde er im dortigen Krankenhaus mit Atropin behandelt und daraufhin zur weiteren Behandlung nach Berlin in die Charité ausgeflogen. Ein Speziallabor der Bundeswehr wies später ein Nervengift der Nowitschok-Gruppe in den in der Charité entnommenen Proben Nawalnys nach. ${ }^{73}$ Der Vorfall fachte sogleich die Diskussion über die Fertigstellung der Ostseepipeline „Nord Stream 2“ wieder an. Die EU verhängte weitere Sanktionen gegen Russland, die am 15. Oktober 2020 in Kraft traten und Einreiseverbote sowie Kontensperrungen für rund zehn Personen und ein Forschungsinstitut umfassen. ${ }^{74}$ Auch die Wirtschaftssanktionen wegen des Ukraine-Konflikts wurden Anfang Dezember um weitere sechs Monate verlängert. ${ }^{75}$ In Reaktion hierauf erließ Russland Sanktionen gegen VertreterInnen Deutschlands und anderer EU-Mitgliedstaaten. ${ }^{76}$ Der Vorfall und die Reaktionen verdeutlichen, wie angespannt die Beziehungen der EU und Deutschlands zu Russland weiter sind. Aufgrund der innerdeutschen und innereuropäischen Differenzen hinsichtlich „Nord Stream 2“ war Deutschland hier nicht in der Lage, eine Vermittlerrolle einzunehmen. So konnten während der deutschen EU-Ratspräsidentschaft keine Fortschritte bei der Verbesserung der EU-Russland-Beziehungen erreicht werden.

69 Rat der Europäischen Union: Schlussfolgerungen des Präsidenten des Europäischen Rates im Anschluss an die Videokonferenz der Mitglieder des Europäischen Rates vom 19. August 2020, Pressemitteilung 542/20, 19. August 2020 .

70 Böttger: After the Presidential Election in Belarus, 2020.

71 Rat der Europäischen Union: Belarus: EU imposes sanctions for repression and election falsification, Pressemitteilung 626/20, 2. Oktober 2020.

72 Tagesschau.de: EU beschließt Sanktionen gegen Belarus, 2. Oktober 2020.

73 Presse- und Informationsamt der Bundesregierung: Erklärung der Bundesregierung im Fall Nawalny, Pressemitteilung 306, 2. September 2020.

74 Durchführungsverordnung (EU) 2020/1480 des Rates vom 14. Oktober 2020 zur Durchführung der Verordnung (EU) 2018/1542 über restriktive Maßnahmen gegen die Verbreitung und den Einsatz chemischer Waffen, in: Amtsblatt der EU, Nr. L 341 vom 15. Oktober 2020, S. 1-6.

75 Tagesschau.de: Weitere EU-Sanktionen gegen Russland und Türkei, 11. Dezember 2020.

76 Zeit Online: Russland kündigt Sanktionen gegen Deutschland an, 12. November 2020; Deutsche Welle Online: Fall Nawalny: Russland antwortet auf EU-Sanktionen, 22. Dezember 2020. 


\section{Die transatlantischen Beziehungen}

Nach Jahren Trumpscher Irritationen im transatlantischen Verhältnis nahmen von der Leyen und Borrell den Wahlsieg Bidens zum Anlass, dem Rat sowie dem Europäischen Rat am 2. Dezember 2020 eine Mitteilung über eine „neue EU-US-Agenda für den globalen Wandel" vorzulegen, in der die Einrichtung eines transatlantischen Handels- und Technologierates, eine Konformitätsbewertung hinsichtlich der beiderseitigen Standards und des Regelungsniveaus, die bessere Kontrolle von Big-Data-Plattformen, ein EU-US-Sicherheits- und Verteidigungsdialog, eine grüne Handelsagenda und die Mitwirkung an einem von Biden ins Spiel gebrachten Gipfel für Demokratie vorgeschlagen wurden. ${ }^{77}$

Der Außenministerrat griff dies in seiner Dezember-Sitzung mit Unterstützung der deutschen Ratspräsidentschaft auf und bekannte sich zur Notwendigkeit einer „erneuerte[n] transatlantische[n] Agenda“, die das Eintreten für die Sustainable Development Goals bis 2030, eine umweltfreundliche Energiepolitik und die Hoffnung auf einen erneuten Beitritt der USA zum Pariser Klimaabkommen beinhaltete. ${ }^{78}$ Auch verband sich damit die Hoffnung auf die Beilegung von Handelsstreitigkeiten, eine bessere Koordinierung von Sanktionen und die Aufhebung der Extraterritorialität amerikanischer Russlandsanktionen, was mit Blick auf die an „Nord Stream 2“ beteiligten Firmen ein besonderes Anliegen der deutschen Ratspräsidentschaft war. Zudem sollten die Vereinten Nationen, die Weltgesundheitsorganisation (WHO), die Welthandelsorganisation (WTO) und die EU-NATO-Beziehungen gestärkt werden. ${ }^{79}$

Der Europäische Rat griff aber die neue transatlantische Agenda auf seiner Tagung am 10. und 11. Dezember 2020 nicht auf und äußerte sich nur allgemein: Er betonte, dass sich die EU auf die Kooperation mit den USA bei der Bekämpfung der COVID-19-Pandemie und ihrer ökonomischen Folgen, der Bewältigung des digitalen Wandels, der Verbesserung der Handelsbeziehungen und der Reform der WTO freue. ${ }^{80}$ Die Staats- und Regierungschefs hielten sich eher zurück, weil das amerikanische Electoral College zur Wahl des neuen Präsidenten erst nach dem europäischen Gipfel am 14. Dezember zusammentraf und die Wahl Bidens noch nicht offiziell erfolgt war. Andererseits stand das EU-China-Investitionsabkommen, das zu einer der großen Prioritäten der deutschen Ratspräsidentschaft gehörte, kurz vor dem Abschluss und es war nicht nur die Bundeskanzlerin, die dies unbedingt noch unter Dach und Fach bringen wollte, bevor die Haltung Bidens gegenüber dem „Systemrivalen China“ offizielle amerikanische Außenpolitik wurde.

\section{Die EU-China-Beziehungen}

Auch die EU-China-Beziehungen waren ein wichtiger Schwerpunkt der deutschen EURatspräsidentschaft, da China der bedeutendste Markt für die nationale Außenwirtschaft

77 Europäische Kommission/Hoher Vertreter der Union für Außen- und Sicherheitspolitik: Gemeinsame Mitteilung an das Europäische Parlament, den Europäischen Rat und den Rat. Eine neue EU-US-Agenda für den globalen Wandel, JOIN(2020) 22 final.

78 Rat der Europäischen Union: Schlussfolgerungen des Rates zu den Beziehungen zwischen der Europäischen Union und den Vereinigten Staaten, Pressemitteilung 884/20, 7. Dezember 2020.

79 Ebenda; vgl. auch Bettina Klein: Zeit für eine neue transatlantische Agenda, in: Deutschlandfunk online, 7. Dezember 2020; Moritz Koch: Europa und die USA - Eine mühsame Annäherung, in: Handelsblatt, 18. August 2020; sowie Georg Blume: Das schmeckt nach mehr, in: Zeit Online, 16. Dezember 2020.

80 Europäischer Rat: Schlussfolgerungen, Dezember 2020, EUCO 22/20, Ziff. 29. 
ist. Der geplante Gipfel mit China und allen 27 Staats- und Regierungschefs musste allerdings aufgrund der COVID-19-Pandemie verschoben werden. Im Vorfeld der deutschen EU-Ratspräsidentschaft nahm das europäisch-chinesische Verhältnis einen besonderen Stellenwert ein. Insbesondere sah Kanzlerin Merkel trotz großer weltwirtschaftlicher Bedeutung Chinas die Notwendigkeit eines selbstbewussten und entschlossenen Auftretens gegenüber der chinesischen Regierung. ${ }^{81}$ Die Beziehungen zwischen China und der EU sind ambivalent. Dies zeigt die Einordnung Chinas durch die Europäische Kommission im März 2019 als „strategischen Partner“, „wirtschaftlichen Konkurrenten“ und „Systemrivalen" ${ }^{82}$ Neben der Sicherheits- und Handelspolitik wird in der Klimapolitik die Notwendigkeit einer engen Kooperation mit China unterstrichen. Hier zeigten sich jedoch wiederholt Konflikte über restriktive Investitionsregelungen und Marktzugänge für europäische Firmen. Diese Streitpunkte sollten unter deutscher Ratspräsidentschaft geklärt und beim EUChina-Gipfel in Leipzig im September 2020 ein EU-China-Investitionsabkommen geschlossen werden. Der Gipfel musste in viel kleinerem Rahmen virtuell durchgeführt werden. Angesichts einer Vielzahl von Konflikten waren die Chancen auf einen Erfolg allerdings schon vor Ausbruch der COVID-19-Pandemie als eher schlecht eingeschätzt worden. Zudem wurde die Abhängigkeit von chinesischen Lieferketten durch die Pandemie zunehmend kritisch bewertet. Am 30. Dezember 2020 einigten sich die EU und China in abschließenden Verhandlungen auf höchster Ebene nach sieben Jahren Verhandlung schließlich doch noch auf ein Investitionsabkommen, das die Wettbewerbsbedingungen und den Zugang für europäische Investitionen in China ohne Joint-Venture-Zwang verbessern soll. ${ }^{83}$ Streitthema waren bis zuletzt die Arbeitsrechte, bei denen China nun weitere Anstrengungen zur Umsetzung der Richtlinien der Internationalen Arbeitsorganisation (ILO) verspricht - ein Kompromiss, der manchen nicht weit genug geht. ${ }^{84}$

\section{Eine erste Bilanz der deutschen Ratspräsidentschaft}

Zwei weitere Politikbereiche, die zum Programm der deutschen EU-Ratspräsidentschaft gehörten, waren die Klimapolitik und die Konferenz zur Zukunft Europas. Erstere fand ihren Niederschlag in der Festlegung bei der Mittelverwendung des MFR wie auch des Aufbaufonds zu 30 Prozent für die Klimaschutzziele. Der deutschen Ratspräsidentschaft gelang darüber hinaus, einen weiteren Kompromiss in letzter Minute zu finden: Die Treibhausgasemissionen in der EU sollen bis 2030 statt um 40 nun um 55 Prozent gesenkt

81 Die Bundeskanzlerin: Rede von Bundeskanzlerin Merkel im Rahmen der Veranstaltung „Außen- und Sicherheitspolitik in der deutschen EU-Ratspräsidentschaft“ der Konrad-Adenauer-Stiftung am 27. Mai 2020, abrufbar unter: https://www.bundeskanzlerin.de/bkin-de/aktuelles/rede-von-bundeskanzlerin-merkel-im-rahmender-veranstaltung-aussen-und-sicherheitspolitik-in-der-deutschen-eu-ratspraesidentschaft-der-konrad-adena uer-stiftung-am-27-mai-2020-1755884 (letzter Zugriff: 7.10.2020).

82 Europäische Kommission/Hoher Vertreter der Union für Außen- und Sicherheitspolitik: Gemeinsame Mitteilung an das Europäische Parlament, den Europäischen Rat und den Rat. EU-China - Strategische Perspektiven, JOIN(2019) 5 final.

83 Zeit Online: EU und China einigen sich auf Investitionsabkommen, 30. Dezember 2020.

84 Reinhard Bütikofer: Verhandlungsergebnis des EU-China Investitionsabkommens, Pressemitteilung, 29. Dezember 2020, abrufbar unter: https://reinhardbuetikofer.eu/2020/12/29/verhandlungsergebnis-der-eu-china-i nvestitionsabkommens-i-pressemitteilung/ (letzter Zugriff: 28.1.2021); Nicole Bastian/Dana Heide/Moritz Koch/Hans-Peter Siebenhaar/Frank Specht/Klaus Stratmann: Der Deal steht: Bei welchen Fragen sich die EU durchgesetzt hat - und wo China, in: Handelsblatt, 30. Dezember 2020. 
werden. ${ }^{85}$ Dies könnte einen nicht unbedeutenden Durchbruch in Richtung $\mathrm{CO}_{2}$-Reduktion bedeuten.

Die für die weitere Entwicklung der EU-Integration wichtige Konferenz zur Zukunft Europas, ${ }^{86}$ die Antworten für die Zukunft der europäischen Demokratie formulieren und die nächsten Schritte der europäischen Integration vorzeichnen soll, ${ }^{87}$ muss hingegen weiterhin auf ihren Auftakt warten. Sie war Teil des Regierungsprogramms der damals designierten Kommissionspräsidentin von der Leyen und sollte am Europatag, dem 9. Mai 2020, eröffnet werden. Jedoch konnte bis zum Ende der deutschen Ratspräsidentschaft keine Einigung auf einen Vorsitz erzielt werden, sodass sich der Beginn weiter verzögerte. ${ }^{88}$

Insgesamt hat die Ratspräsidentschaft trotz einer "gemischten Bilanz" ${ }^{\text {" }}$ durch einige spektakuläre Erfolge bei den Verhandlungen über den MFR einschließlich NGEU-Fonds, über den Brexit und in letzter Minute über das EU-China-Abkommen die Erwartungen übertroffen. Hierbei hat die Bundesregierung in vielen Fällen ihre Vermittlerrolle als EU-Ratspräsidentin in den Vordergrund gestellt, um - so beim MFR und dem Rechtsstaatsmechanismus - realisierbare Kompromisse zu finden. Dabei hat sie inhaltlich ihre eigene Handschrift hinterlassen können, denn viele der Bereiche, in denen sie die EU vorangebracht hat, beziehen sich auf Finanzfragen und Handelsvereinbarungen, die für Deutschland als größten Nettozahler der EU und als Exportnation besonders wichtig sind. Das betrifft in letztgenannter Hinsicht vor allem das Handelsabkommen mit dem Vereinigten Königreich und das Investitionsabkommen mit China sowie in erstgenannter Hinsicht das "Megafinanzpaket“ der EU, bei dem ein direkter Weg in eine Schuldenunion durch die Absicherung über den Eigenmittelbeschluss und Rückzahlungsmodalitäten verhindert wurde.

Dennoch bleibt die deutsche Kehrtwende hin zur begrenzten und zeitlich befristeten gemeinschaftlichen Mittelaufnahme am Kapitalmarkt äußerst bemerkenswert. Wie war ein solcher selektiver Supranationalisierungsschub mit einer gestärkten Rolle der Kommission möglich? Zum einen war der deutsche Finanzminister Olaf Scholz im Prinzip für kreditfinanzierte nationale und europäische Konjunkturprogramme (ganz anders noch als Wolfgang Schäuble als Finanzminister). Zum anderen ließen sich die wirtschaftlichen Folgen der Corona-Krise nicht auf ein Selbstverschulden einzelner Mitgliedstaaten durch ein „Leben über ihre Verhältnisse“ (so der Vorwurf der nördlichen gegenüber den südlichen Mitgliedstaaten während der Finanzkrise in der Eurozone) zurückführen. Die Corona-Krise betrifft alle Mitgliedstaaten gleichermaßen. Des Weiteren hatte sich schon in der Eurokrise gezeigt, dass Deutschland am Ende den Euro und das Funktionieren des Binnenmarktes absichern musste, um nicht hohe Verluste im deutschen Export zu erleben, der zu fast 60 Prozent in den EU-Binnenmarkt geht. ${ }^{90}$ Nun war aber nicht nur die

85 Europäischer Rat: Schlussfolgerungen, Dezember 2020, EUCO 22/20, Ziff. 12 und 15.

86 Julian Plottka: Die Konferenz zur Zukunft Europas zwischen „Konvent 2.0“ und „Intergouvernementalismus 3.0“: Warum Europa diese Chance zur Reform nutzen muss, in: integration 3/2020, S. 231-244.

87 Ursula von der Leyen: Eine Union, die mehr erreichen will. Meine Agenda für Europa. Politische Leitlinien für die künftige Europäische Kommission 2019-2024.

88 Spiegel Online: Streit über Vorsitz blockiert Beginn der EU-Zukunftskonferenz, 1. Dezember 2020.

89 Bernd Riegert: EU: Gemischte Bilanz der deutschen „Corona-Präsidentschaft“, in: Deutsche Welle online, 30. Dezember 2020.

90 Christian Dreger: Der wirtschaftliche Nutzen Europas für Deutschland, in: Katrin Böttger/Mathias Jopp (Hrsg.): Handbuch zur deutschen Europapolitik, Baden-Baden 2021, S. 89-103. 
europäische Wirtschaft von einem tiefen Einbruch bedroht, sondern die Weltwirtschaft, sodass wenigstens die Stützung des europäischen Marktes erreicht werden musste. Auch hilft ein EU-Finanzpaket, eine überproportionale Zunahme der Staatsverschuldung der Problemstaaten des Südens zu verhindern. Zudem galt es, die Wunden der Eurokrise, die in den Beziehungen zwischen Deutschland und Italien entstanden waren, zu heilen ${ }^{91}$ und den Schulterschluss mit Frankreich als wichtigstem und verlässlichstem Partner in der EU zu suchen, um Existenz und Handlungsfähigkeit der EU abzusichern. Alle diese Aspekte erklären den Schritt hin zu einem fiskalischen Föderalismus in der Krise durch Schaffung eines zentralen finanziellen EU-Interventionsinstruments, wenn auch in begrenzter Höhe und zeitlich befristetet.

Weitere drängende Themen wie die EU-Afrika-Beziehungen, die Digital-, Bildungsund Gesundheitspolitik, die EU-Indien-Beziehungen und der Beginn der Konferenz zur Zukunft Europas wurden von der deutschen Ratspräsidentschaft nicht mehr aufgegriffen, sondern der portugiesischen überlassen. ${ }^{92}$ Insbesondere die Krise der EU-Flüchtlingsund Migrationspolitik, die durch das Feuer im Lager Moria verstärkt in das Licht der Öffentlichkeit gerückt wurde, gehört wohl zu den größten „Leftovers“ der deutschen Ratspräsidentschaft. Hierbei handelt es sich allerdings grundsätzlich um einen schwierigen Politikbereich, was sich auch am Scheitern des Vorschlags der Kommission zum Asylund Migrationspaket an der mangelnden Zustimmung der Mitgliedstaaten im September 2020 zeigte. ${ }^{93}$ Im Jahr 2021 wird es auch mit Blick auf den legislativen Zyklus vor allem aber erst einmal um die rechtliche und praktische Umsetzung zahlreicher Beschlüsse gehen. So müssen der MFR und der Wiederaufbaufonds erst noch von allen nationalen Parlamenten ratifiziert werden. Das Rechtsstaatsinstrument wird vorläufig nicht mit Leben gefüllt werden, denn Ungarn und Polen wollen gegen die Verordnung vor dem EuGH klagen, wodurch es zu Verzögerungen kommen kann. Auch das Handelsabkommen mit dem Vereinigten Königreich oder das Investitionsabkommen mit China müssen in die Praxis umgesetzt werden, wobei sicher noch die eine oder andere Hürde zu nehmen sein wird. Dass die deutsche Ratspräsidentschaft überhaupt viel bewegen konnte, lag teilweise an der Schwäche vorausgegangener Präsidentschaften, den Unsicherheiten des neuen Führungspersonals der europäischen Institutionen und dem „Selbstfindungsprozess“ des Europäischen Parlaments, wo Entscheidungen nicht mehr durch eine mehrheitsfähige große Koalition zwischen Christdemokraten und Sozialisten prädefiniert werden können. Das alles hat zu einem Problemstau bei wichtigen Themen geführt, bei denen Deutschland durch Erfahrung, geschickte Verhandlungsführung und Durchsetzungskraft als größter EU-Mitgliedstaat sowie unter dem Druck der dringend benötigten Einigung über die Finanzierung der kommenden Jahre den gordischen Knoten durchschlagen konnte. Das integrationspolitisch Interessante dabei sind die schon in anderen Krisen feststellbaren

91 Vgl. hierzu die Ausführungen von Gabriele D’Ottavio: Anomalie oder Laboratorium? Italien und der „antideutsche Euroskeptizismus“, in: Katrin Böttger/Mathias Jopp (Hrsg.): Handbuch zur deutschen Europapolitik, Baden-Baden 2021, S. 527-544.

92 Oliver Neuroth: Portugal übernimmt Ratspräsidentschaft, in: Tagesschau.de, 1. Januar 2021; euronews: EURatspräsidentschaft: Große Aufgaben für Portugal, 5. Januar 2021; siehe hierzu auch Minna Ålander: Die portugiesische EU-Ratspräsidentschaft: Budget, Brexit, Biden und andere Herausforderungen, in diesem Heft, S. 71-78.

93 Siehe hierzu Florian Trauner: Das neue EU-Migrations- und Asylpaket: Wird es zu mehr Solidarität zwischen den Mitgliedstaaten führen?, in diesem Heft, S. 40-52. 
Schübe in Richtung einer Zentralisierung wichtiger Aufgaben auf der Brüsseler Ebene, einhergehend mit einer Stärkung der supranationalen Institutionen. ${ }^{94} \mathrm{Ob}$ das Beispiel von NGEU als kreditfinanziertes antizyklisches EU-Instrument Schule macht oder einmalig und begrenzt bleibt, wird sich erst noch erweisen müssen. Jedenfalls hat sich die deutsche Ratspräsidentschaft nicht nur zum wiederholten Male als Problemlöser erwiesen, sondern auch eine neue Tür an Möglichkeiten der integrationspolitischen Vertiefung aufgestoßen.

94 Renaud Dehousse: Why has EU macroeconomic governance become more supranational?, in: Journal of European Integration 5/2016, S. 617-631. 